\title{
Diagnóstico de enfermería para los pacientes con tuberculosis en la unidad de enfermedades infecciosas
}

\section{Nursing diagnoses for patients with tuberculosis in the infectious diseases unit \\ Diagnósticos de enfermagem para pacientes com tuberculose na unidade de infectologia}

\author{
Vinícius Lino de Souza Neto ${ }^{1}$, Richardson Augusto Rosendo da \\ Silva², Ivana de Araújo Fonseca ${ }^{3}$, Sergilene Fonseca Teixeira \\ Santos ${ }^{4}$, Anzielyson Felix Silva dos Santos ${ }^{5}$, Ana Gláucia Lopes do \\ Nascimento $^{6}$ \\ ${ }^{1}$ Enfermeiro, Mestre em Enfermagem pela Universidade Federal do Rio Grande \\ do Norte - UFRN; Professor do curso Bacharelado em Enfermagem \\ Universidade Federal do Rio Grande do Norte/UFRN e Faculdade Natalense de \\ Ensino e Cultura - FANEC. Natal/RN, Brasil. Correo electrónico: \\ vinolino@hotmail.com \\ ${ }^{2}$ Enfermeiro, Doutor, Docente, Universidade Federal do Rio Grande do \\ Norte/UFRN. Natal, Rio Grande do Norte (RN), Brasil. Correo electrónico: \\ rirosendo@hotmail.com \\ ${ }^{3}$ Discente, Graduação em Enfermagem, Universidade Federal do Rio Grande do \\ Norte/UFRN. Natal, Rio Grande do Norte (RN), Brasil. Correo electrónico: \\ ivanafonsecaa@hotmail.com \\ ${ }^{4}$ Discente, Graduação em Enfermagem, Faculdade Natalense de Ensino e \\ Cultura - FANEC.Correo electrónico: sergiilene.100@hotmail.com \\ ${ }^{5}$ Discente, Graduação em Enfermagem, Faculdade Natalense de Ensino e \\ Cultura - FANEC.Correo electrónico: anzielyson.santos@gmail.com \\ ${ }^{6}$ Discente, Graduação em Enfermagem, Faculdade Natalense de Ensino e \\ Cultura-FANEC. Correo electrónico: glaucia@colegiodasneves.com.br
}

Cómo citar este artículo en edición digital: Neto, V. L. S., Silva, R.A.R., Fonseca, I. A., Santos, S. F. T., dos Santos, A. F. S., \& Nascimento, A. G. L. (2019). Diagnóstico de enfermería para los pacientes con tuberculosis en la unidad de enfermedades infecciosas. Cultura de los Cuidados (Edición digital), 23

(54). Recuperado de http://dx.doi.org/10.14198/cuid.2019.54.29

Correspondencia: Vinicius Lino de Souza Neto.Universidade Federal do Rio Grande do Norte, Centro de Ciências da Saúde - Departamento de Enfermagem. Campus Central, s/n, Lagoa Nova 59078-970 Natal, Rio Grande do Norte (RN)

Correo electrónico de contacto: vinolino@hotmail.com Recibido: 20/01/2018; Aceptado: 11/04/2019

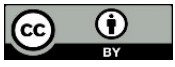

\begin{abstract}
Objective: To list the nursing diagnoses in individuals with tuberculosis in an inpatient unit in infectious diseases.
\end{abstract}

Method: This is a case study developed with 02 patients in a Infectious Diseases inpatient unit in a teaching hospital, 
reference for the treatment of infectious diseases, in the Northeast of Brazil. For data collection was used a structured questionnaire was developed by the researchers on the theory of basic human needs of Wanda Horta. Then the diagnostic inferences were formulated, and so outlined in concept maps. The CIPE®2015 was the terminology used, thus following the principles established by the International Council of Nurses (ICN).

Results: The findings allowed the identification of the following prevalent diagnosis in patients with tuberculosis: peripheral edema, impaired dietary nutrition, hygiene impaired oral cavity hygiene standard compromised scalp, insomnia, tobacco abuse, low self-esteem and social isolation.

Conclusions: Patients exhibited nursing diagnoses related to changes in psychobiological and psychosocial needs, which requires action planning and specific care.

Keywords: Tuberculosis, communicable diseases, nursing process, nursing care.

\section{RESUMO}

Objetivo: Elencar os diagnósticos de enfermagem em indivíduos com tuberculose em uma unidade de internação em infectologia.

Método: Trata-se de um estudo de caso desenvolvido com 02 pacientes em uma unidade de internação de infectologia em um Hospital de Ensino, referência para o tratamento de doenças infectocontagiosas, no Nordeste do Brasil. Para a coleta de dados utilizou-se um formulário estruturado, elaborado pelos pesquisadores seguindo a teoria das necessidades humanas básicas de Wanda Horta. Em seguida, formularam-se as inferências diagnósticas, $\mathrm{e}$ assim esquematizados em mapas conceituais. A CIPE® versão 2015 foi a terminologia utilizada, seguindo assim, os preceitos estabelecidos pelo Conselho Internacional de Enfermeiros (ICN).

Resultados: Os achados possibilitaram a identificação dos seguintes diagnósticos prevalentes em pacientes com tuberculose: edema periférico, nutrição dietética prejudicada, higiene da cavidade oral prejudicada, padrão de higiene do couro cabeludo comprometida, insônia, abuso de tabaco, baixa autoestima e isolamento social.

Conclusões: Os pacientes avaliados apresentaram diagnósticos de enfermagem relacionados às alterações das necessidades psicobiológicas e psicossociais, o que requer o planejamento de ações e cuidados específicos.

Palavras chave: Doenças Transmissíveis, tuberculose, processos de enfermagem, cuidados de enfermagem.

\section{RESUMEN}

Objetivo: Elaborar una lista de diagnósticos de enfermería en personas con tuberculosis en una unidad de hospitalización de las enfermedades infecciosas.

Método: Es un estudio de caso desarrollado con 02 pacientes en una unidad de hospitalización Enfermedades Infecciosas en un hospital universitario, de referencia para el tratamiento de enfermedades infecciosas, en el noreste de Brasil. Para la recolección de datos se utilizó un cuestionario estructurado fue desarrollado por los investigadores de la teoría de las necesidades humanas básicas de Wanda Horta. Entonces las inferencias diagnósticas fueron formuladas, y así delineados en los mapas conceptuales. El CIPE® verseo 2015 era la terminología utilizada, siguiendo así los principios 
establecidos por el Consejo Internacional de Enfermeras (CIE).

Resultados: Los hallazgos permitieron la identificación de los siguientes diagnósticos prevalentes en pacientes con tuberculosis: edema periférico, problemas de nutrición dietética, higiene deficiente nivel de higiene cavidad oral cuero cabelludo comprometida, el insomnio, el tabaquismo, la baja autoestima y aislamiento social.

Conclusiones: Los pacientes presentaron diagnósticos de enfermería relacionados con los cambios en las necesidades psicobiológicas y psicosociales, lo que requiere la planificación de acciones y cuidados específicos.

Palabras clave: Tuberculosis, enfermedades transmisibles, procesos de enfermería, atención de enfermería.

\section{INTRODUÇÃO}

A tuberculose configura-se como uma doença de alta incidência nos diversos níveis sociais, acometendo pessoas em situações de vulnerabilidade, constituindo assim um grave problema de saúde pública. Apesar de ter tratamento e cura, os índices de morbimortalidade vêm crescendo e sua erradicação está longe de ser alcançada em países no processo de desenvolvimento (Brasil, 2012).

Estima-se que no Mundo, em 2012, ocorreram cerca de 8,6 milhões de novos casos de TB e 1,3 milhões de mortes por esta doença. No Brasil, em 2013, foram diagnosticados aproximadamente $71 \mathrm{mil}$ casos novos e 4,6 mil mortes em decorrência da mesma. O país ocupa o $17^{\circ}$ lugar entre os 22 países responsáveis por $80 \%$ do total de casos de TB no mundo, e este índice está diretamente associado com a pobreza, tornando os indígenas, as pessoas privadas de liberdade, os portadores de HIV/AIDS e os moradores de rua mais susceptíveis a doença (Brasil, 2013).

Considerando as regiões do Brasil, verificou-se que no Norte a incidência foi de 45,2/100.000 habitantes, no Sudeste 37,1, no Nordeste 34,7, no Sul 31,3 e no Centro-Oeste 24,6 . No entanto a região que apresentou maior coeficiente de mortalidade foi o Nordeste com 2,6 mortes por cada 100.000 habitantes. Do mesmo modo, o estado da Paraíba também apresentou grande número de casos de TB. No ano de 2013, o coeficiente de incidência da doença foi 28,2/100.000 habitantes e o coeficiente de mortalidade 1,9/100.000 habitantes. Além disso, as taxas de cura $(64,6 \%)$ e de abandono do tratamento $(13,0 \%)$ estão superiores às recomendadas pela Organização Mundial de Saúde (OMS), que preconiza um percentual de cura superior a $85 \%$ dos casos detectados e redução da taxa de abandono a menos de 5\% dos casos (Brasil, 2013).

Neste cenário, os profissionais de enfermagem são constantemente desafiados na busca de conhecimento técnico-científico frente aos indivíduos acometidos por $\mathrm{TB}$, a fim de promover a melhoria do cuidado prestado ao cliente, focado nas necessidades de cuidado do indivíduo, família e comunidade (Bittencourt \& Crossetti, 2013).

Dentre os vários métodos científicos assistências usadas pelos profissionais de enfermagem, estão às práticas sistemáticas. Estas se referem a uma sequência de etapas específicas, como identificação das condições de saúde que requerem intervenções, planejamento, implementação e avaliação das ações de enfermagem. Todos estes mecanismos têm como objetivo proporcionar atendimento profissional ao cliente, seja indivíduo, família ou comunidade, considerando suas singularidades (Alves, Lopes, Jorge, 2010). 
Com esse entendimento a operacionalização de ações de enfermagem de forma sistematizada, torna-se fundamental a utilização de uma terminologia comum, pois a documentação do cuidado, conforme o Processo de enfermagem é viabilizado por meio da utilização de terminologias. Dentre as mais conhecidas no Brasil, que apoiam o PE, encontram-se a NANDA Internacional (North American Nursing Diagnosis Association), a NIC (Nursing Interventions Classification), a NOC (Nursing Outcomes Classification), a CIPE (Classificação Internacional para a Prática de Enfermagem) e o inventário vocabular da CIPESC (Classificação Internacional das Práticas de Enfermagem em Saúde Coletiva) (Alves, Lopes, Jorge, 2010).

A justificativa para o desenvolvimento do estudo se deu pelo fato de existir um numero reduzido de produções científicas, principalmente no âmbito de infectologia, que retrate os diagnósticos de enfermagem aos pacientes com tuberculose. Para nortear o desenvolvimento do presente estudo, formulou-se a seguinte questão norteadora: Quais os diagnósticos de enfermagem á pacientes com tuberculose á partir da $\mathrm{CIPE}^{\circledR}$ 2.0? Neste sentido o estudo teve como objetivo elencar os diagnósticos de enfermagem para pacientes com tuberculose hospitalizados em uma unidade de infectologia.

A relevância do estudo é contribuir para a comunidade cientifica através dos achados diagnóstico para pessoas com tuberculose e implementar uma assistência qualificada baseada em evidencias, correspondendo a necessidades humanas básicas do individuo.

\section{METODO}

Trata-se de um estudo de caso desenvolvido com 02 pacientes em uma unidade de internação de infectologia em um Hospital de Ensino, referência para o tratamento de doenças infectocontagiosas, no Nordeste do Brasil. Para a seleção dos sujeitos da pesquisa foram adotados os seguintes critérios: estar hospitalizado na clínica no momento da coleta de dados; idade maior que 18 anos; ter como diagnóstico médico tuberculose primária. Foram excluídos do estudo os pacientes que apresentarem: como causa da tuberculose a co-infecção por HIV/AIDS.

Os dados foram coletados em fevereiro de 2014 por meio de roteiro de entrevista e exame físico, embasado pelo modelo conceitual de Horta, categorizado conforme as Necessidades Humanas Básicas, sendo subdividido em psicobiológicas, psicossociais e psicoespirituais.

$\mathrm{Na}$ entrevista, buscou-se a idade, procedência, sexo, diagnóstico. Ao exame físico utilizaram-se as técnicas propedêuticas inspeção, percussão, palpação e ausculta, e complementando o processo foram utilizados os seguintes equipamentos: um oxímetro de pulso portátil, para a mensuração da saturação do nível de oxigênio, um esfigmomanometro e estetoscópio para verificar a pressão arterial, e por fim um termômetro, a fim de verificar a curva térmica.

Após as coletas dos dados, elencaram-se os fenômenos da prática de enfermagem, catalogando-se conforme as necessidades psicobiológicas, psicossociais e espirituais. E assim formulou-se os diagnósticos de enfermagem, baseados no desenvolvimento do raciocínio diagnóstico e/ou julgamento clínico, diante das necessidades do indivíduo, e esquematizados em mapas conceituais para uma melhor compreensão 
do raciocínio diagnóstico na construção das afirmativas.

Com isso, utilizou-se a CIPE ${ }^{\circledR}$ versão 2015 como banco terminológico na referida ação, seguindo as normativas estabelecidas pelo Conselho Internacional de Enfermeiros (ICN). Para a devida construção dos diagnósticos de enfermagem, os preceitos interposto pelo presente conselho retratam que a construção das afirmativas de diagnósticos deve ser composta por um termo do eixo foco e julgamento. Antes de iniciar a coleta de dados, os participantes do estudo foram esclarecidos acerca do objetivo da pesquisa, e foi realizada uma leitura do Termo de Consentimento Livre e Esclarecido (TCLE).

O projeto foi aprovado pelo Comitê de Ética em Pesquisa Assim, a pesquisa foi conduzida de acordo com a Resolução nº 466/12 do Conselho Nacional de Saúde, e a Resolução COFEN no 311/2007, que infere sobre o Código de Ética dos Profissionais de Enfermagem.

\section{RESULTADOS}

Neste sentido, na caracterização sociodemográficas, os indivíduos da amostra apresentavam uma faixa etária de 51 á 58 anos, sexo masculino, solteiro, tendo como função laboral carpinteiro e o outro agricultor, com diagnóstico fechado de tuberculose pulmonar para ambos.

Logo após o agrupamento dos dados sociodemográficos, aliaram-se os dados das necessidades humanas básicas em formato de caso clínico que foram exemplificados em mapas conceituais. A seguir, os dados dos pacientes e seus respectivos mapas conceituais Figura 1 e 2:

\section{Apresentação dos casos:}

\# J. S. L, 51 anos, sexo masculino, casado, agricultor, TB, Fr: 18 ipm, Saturação de O2 (Spo2): 99 \%, edema em Membros Inferiores (MMII), Circunferência Abdominal (CA): 81 $\mathrm{cm}$, apetite diminuído, aceita a dieta parcial, Abdômen ( $\mathrm{ABO}$ ): flácido e plano, Peso: 65 $\mathrm{kg}$, A: 1,65 cm, Índice de Massa Corporal (IMC): 23,89 , sono e repouso prejudicado, faz uso de medicação para dormir, deambula, consciente, isocoricas, presença de caspa, turgor normal, T. axilar: $36,5^{\circ} \mathrm{C}$, faz uso de antibioticoterapia, Pulso: $78 \mathrm{bpm}, \mathrm{PA}$ : $120 \times 80 \mathrm{mmhg}$, tabagista há 18 anos, nega etilismo e uso de drogas, analfabeto, medo, apresenta tristeza e tem medo de expor suas ideias.

\#F. C. B, casado, sexo masculino, carpinteiro, TB, 58 anos, FR: 18 irpm, Spo2: $95 \%$, apetite normal com aceite de dieta total, ABO: plano e flácido sem timpanismo, Circunferência Abdominal (CA): $98 \mathrm{~cm}, \mathrm{P}$ : $72 \mathrm{~kg}$, A: 1,78 cm, IMC: 22,78, sono e repouso preservado, halitose, couro cabeludo com caspa, turgor normal, Temperatura axilar 36,3ํㅡ, Fc: 74 bpm, P: 74 bpm, PA: $110 \times 80$ mmhg, nega tabagismo, etilismo e uso de drogas, e falta de auto-confiança. 
FIGURA 1: Mapa conceitual das inferências diagnósticas

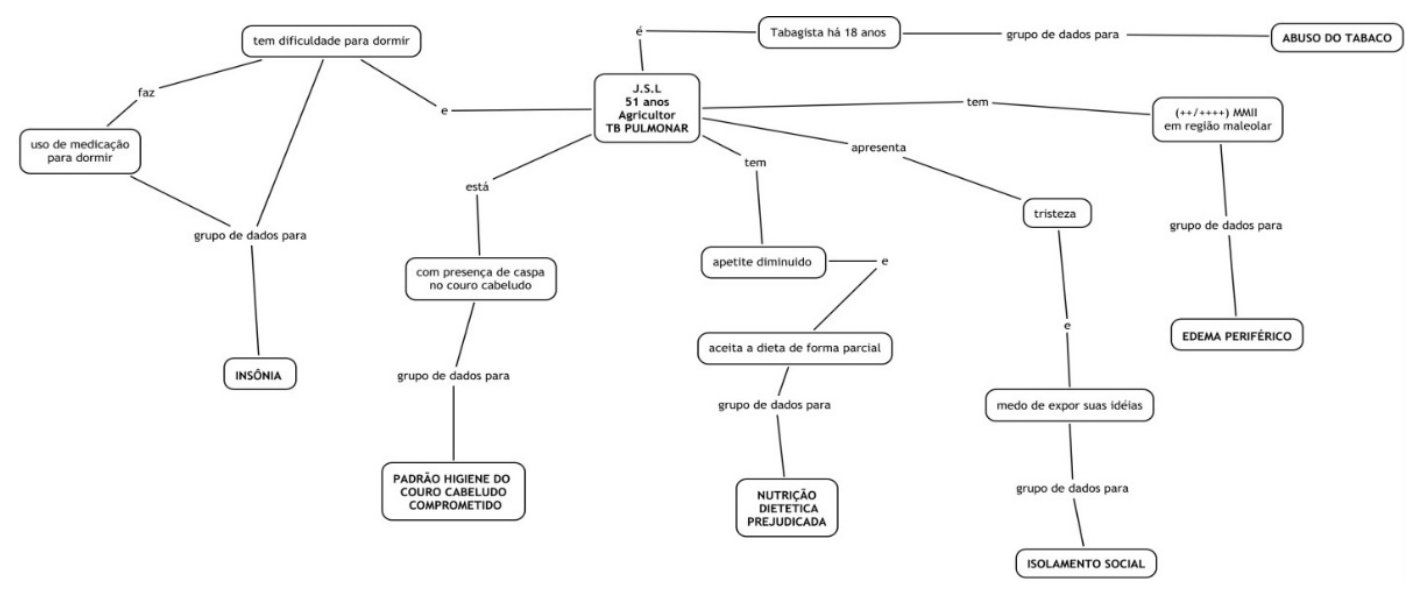

FIGURA 2: Mapa conceitual das inferências diagnósticas

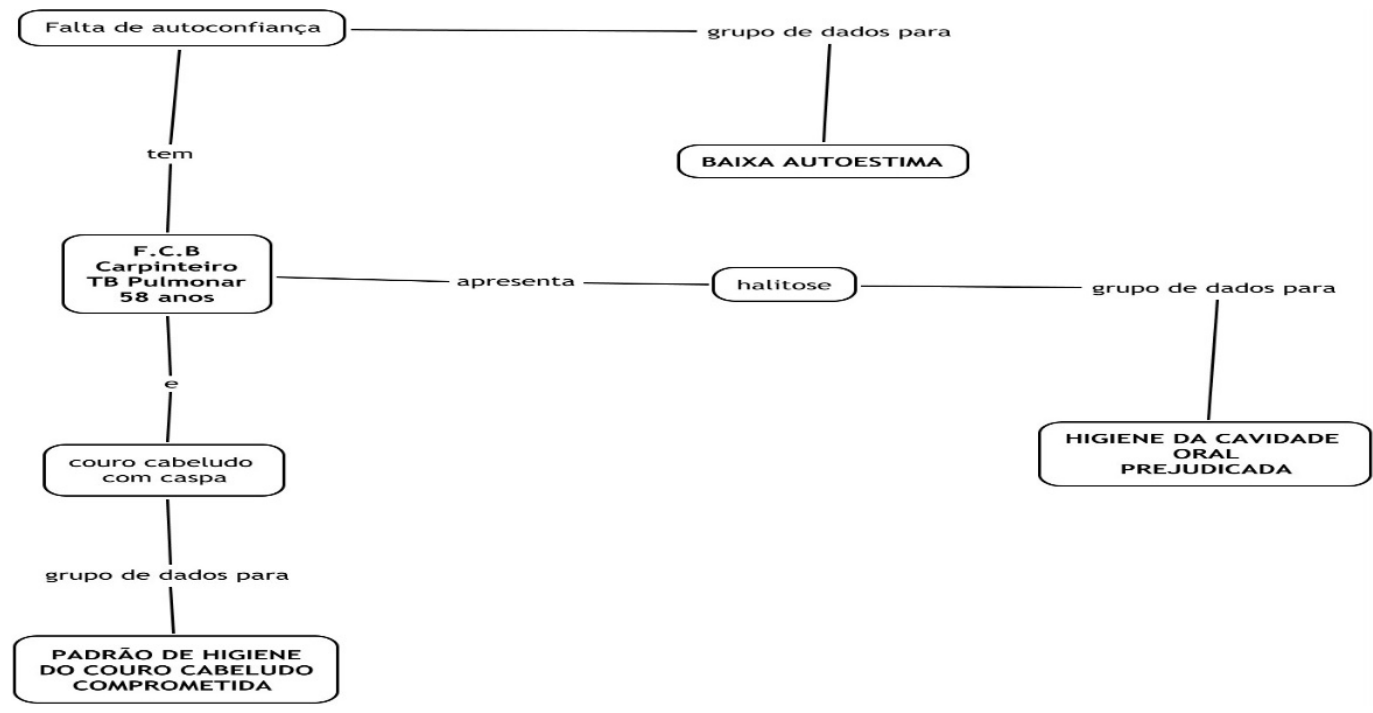


Após a esquematização em mapas conceituais, e para um melhor entendimento dos resultados obtidos, agrupou-se as afirmativas de diagnósticos em tabela de acordo com as necessidades humanas básicas. No total de afirmativas encontradas foram oito, demonstrado logo abaixo, na Tabela 1:

TABELA 1: Demonstrativo de diagnósticos de enfermagem dos pacientes acometidos por tuberculose conforme necessidades humanas básicas psicobiológicas

\begin{tabular}{|c|c|c|c|}
\hline Necessidades Psicobiológicas & Diagnóstico de Enfermagem & $\mathbf{n}$ & $\%$ \\
\hline & Edema Periférico & 01 & 12,5 \\
\hline \multicolumn{4}{|l|}{ Hidratação e regulação eletrolítica } \\
\hline & Nutrição & 01 & 12,5 \\
\hline Nutrição e Eliminação & dietética prejudicada & & \\
\hline Sono e Repouso & Insônia & 01 & 12,5 \\
\hline & Padrão de higiene do couro & 01 & 12,5 \\
\hline \multirow{3}{*}{$\begin{array}{l}\text { Cuidado corporal } \\
\text { cutaneomucosa }\end{array}$} & cabeludo comprometido & & \\
\hline & Higiene da cavidade oral & 01 & 12,5 \\
\hline & prejudicada & & \\
\hline Segurança Física & Abuso do Tabaco & 01 & 12,5 \\
\hline
\end{tabular}

FONTE: Pesquisa Direta

No que tange as necessidades prática de enfermagem, conforme revela a psicossociais foram elencados os respectivos diagnósticos conforme os fenômenos da Tabela 2, logo abaixo. 


\section{TABELA 2: Demonstrativo de diagnósticos de enfermagem dos pacientes acometidos por tuberculose conforme necessidades humanas básicas psicossociais}

\begin{tabular}{|c|c|c|c|c|}
\hline Necessidades Psicossociais & & \multicolumn{3}{|l|}{ Diagnóstico de Enfermagem } \\
\hline & & & $\mathbf{n}$ & $\%$ \\
\hline Aprendizagem (Educação á saúde) & & Isolamento social & 01 & 12,5 \\
\hline Sociabilidade/ Recreação e Lazer & & & & \\
\hline $\begin{array}{l}\text { Autorealização, } \\
\text { Autoimagem }\end{array}$ & e & Baixa autoestima & 01 & 12,5 \\
\hline
\end{tabular}

FONTE: Pesquisa Direta

\section{DISCUSSÃO}

Após a analise os diagnósticos encontrados foram categorizados, conforme ás necessidades psicobiológicas e psicossociais. Destaca-se que, neste estudo, às necessidades psicoespirituais não foram evidenciadas como as demais, decorrentes da limitação de muitos participantes se absterem ao falar sobre religião.

O paciente com tuberculose apresentam alguns comprometimentos no seu estado geral como, febre baixa vespertina com sudorese, inapetência, emagrecimento, e na forma pulmonar da doença, o paciente geralmente tem-se dor torácica e tosse produtiva. Com isso, a tuberculose primária é uma infecção que evolui a partir do foco pulmonar, do ganglionar ou, em decorrência da disseminação hematogênica. A tuberculose de cunho extrapulmonar pode levar o acometimento nos ossos, sistema urogenital, fígado, baço e a forma mais grave miliar, levando ao desenvolvimento de meningite (Sá et al., 2012).

Nesse sentido, em uma associação entre os achados pertinentes na clínica com a sintomatologia por hora discutida na luz da literatura, conforme mencionado acima, os diagnósticos formulados e categorizados conforme as referidas necessidades, sendo apresentados em forma de mapa conceitual, são: Edema periférico, Nutrição dietética prejudicada, Higiene da cavidade oral prejudicada, Padrão de higiene do couro cabeludo comprometida, Insônia, Abuso de Tabaco, Baixa autoestima, Isolamento social.

\section{Hidratação e regulação eletrolítica}

Diante da necessidade à inferência diagnóstica encontrada foi: "Edema periférico". O edema é um estado de acumulação de líquidos entre os espaços, intra ou extracelulares, onde os dispositivos de regulação estão falhos e as necessidades 
eletrolíticas descompensadas, ou seja, as pressões hidrostática, oncótica e coloidosmoticas apresentam-se em desarmonia, decorrente de diversos mecanismos, o mais comum está na ativação do sistema renina-angiotensina-aldosterona. A presença do edema em pacientes com tuberculose possa está vinculada ao presente mecanismo, pois alguns fármacos como efeito colateral ativa o presente sistema.

Desta forma, cabe ao enfermeiro e a equipe assistencial desenvolver intervenções que atenue o edema, como administração de diuréticos, juntamente com a análise hidroeletrolítica, a realização de movimentos ativos e passivos no leito, controle hídrico, e a educação em saúde frente à utilização dos fármacos e seus efeitos. Outra questão está em orientar junto com o setor de nutrição a importância de obter uma alimentação diversificada, mas que não contenha tanto teor hídrico (Araújo, Nóbrega, Garcia, 2013).

\section{Necessidade de Nutrição}

Dentro da necessidade de nutrição elencou-se a inferência "Nutrição dietética prejudicada". A mesma esteve presente na maioria dos pacientes, pois muitos apresentavam mudanças no paladar. Tudo isso, em decorrência, do uso de medicamentos diários, as papilas gustativas perdem a sua sensibilidade sináptica acarretando uma inapetência alimentar. Por isso, a prática da alimentação diversificada, rica em proteínas, vitaminas, minerais, carboidratos proporciona um melhor aporte nutricional para as devidas necessidades e supre as necessidades metabólicas dos pacientes (Ferraz, Viriato, Moura, 2013).

O prejuízo no mecanismo da deglutição é uma das alterações mais comuns nas pessoas que vivem com tuberculose, em decorrência do excesso de medicamentos que inibem a produção de grelina (hormônio da fome), ou, em decorrência da produção excessiva de citocinas, que inibem a ingestão alimentar (Jasen et al., 2013). Diante disto, o enfermeiro deverá estabelecer prescrições que visem atenuar os sintomas da inapetência, melhorando assim, as condições fisiológicas do paciente, tais como: mensuração do IMC, solicitação de avaliação nutricional, orientação quanto aos hábitos alimentares saudáveis e alimentos irritantes.

\section{Necessidade de Sono e Repouso}

Neste segmento de necessidade elencou-se a inferência diagnóstica: "Insônia". Pacientes com doenças crônicas, geralmente, fazem uso contínuo de medicamentos e algumas drogas apresentam como efeito adverso alterações no padrão do sono, podendo oscilar entre a insônia e a sonolência. Desta forma, dois fatores que alteram o padrão do sono são a mudança de ambiente e as modificações no cardápio alimentar. Entretanto, percebe-se que os quadros de insônia e sonolência levam ao sono e repouso comprometido (Maciel et al., 2010).

No entanto, cabe à equipe assistencial de enfermagem, modular ações que sejam atenuadoras, como: colocar o quarto em penumbra; ofertar alimentos e chás que estimulem o sono, mas que não ocorram interação com a medicação prescrita, ficando a cargo do enfermeiro essa vigilância; realizar massagens relaxantes e encorajar banho tépido antes de dormir, pois é responsável pela liberação de endorfina, um neurotransmissor que proporciona o relaxamento muscular por via endógena. 


\section{Integridade cutaneomucosa e Cuidado corporal}

Diante da presente necessidade, encontraram-se os respectivos diagnósticos: "Padrão de higiene do couro cabeludo comprometido" e "Higiene oral prejudica". Pacientes com tuberculose podem apresentar algumas modificações na pele, couro cabeludo e cavidade oral. A higiene oral, íntima e do couro cabeludo também fazem parte do cuidado corporal, pois muitos pacientes imunodeprimidos apresentam seborreia excessiva no couro cabeludo, m decorrência da produção de liposinas formuladas no folículo piloso. Com isso necessita-se de uma prática higiênica intensiva, com atenção para a fricção do couro cabeludo, a fim de estimular a circulação e melhorar a nutrição do epitélio (Andrade et al., 2013).

A higiene corporal é um mecanismo de profilaxia, principalmente, para os indivíduos que sofrem com as modificações na pele decorrente de uma determinada enfermidade, seja transmissível, ou não. Á prática da higiene corporal proporcionam ao individuo benefícios, como a manutenção da saúde, segurança e bem-estar. O enfermeiro deve orientar aos pacientes sobre a importância da prática e de ser realizada diariamente Diagnósticos de enfermagem para clientes hospitalizados em uma clínica de doenças infectocontagiosas (Faria \& Silva, 2013).

\section{Necessidade de segurança física e ambiente}

Neste interim, frente a necessidade elencou-se o diagnóstico de enfermagem, "Abuso do Tabaco". O uso incorreto dessa substância altera o estado de ânimo ou comportamento do usuário. As drogas lícitas, como o tabaco altera a viscosidade do sangue e liberam bradicinas, potentes vasoconstrictores. O álcool leva a perda do juízo critico concentração e consciência. Já, as drogas ilícitas, como a cocaína e maconha, acarretam a uma exacerbação sintomatológica como taquicardia, convulsão, arritmias ventriculares e alucinações. Assim, as pessoas que abusam desses dois tipos de drogas tornam-se mais vulneráveis para contrair doenças infectocontagiosas (Silva et al., 2013).

\section{NECESSIDADES PSICOSSOCIAIS}

\section{Aprendizagem/Sociabilidade/ Recreação e Lazer}

O Isolamento social foi o diagnóstico de enfermagem encontrado frente à necessidade psicossocial. Á prática do convívio social entre as pessoas com doenças transmissíveis torna-se bastante restritiva, á partir do momento em que o meio social sabe do seu da sua condição de saúde, ou, mesmo não detendo a ciência do fato, o indivíduo tem como mecanismo de defesa o isolamento social, evitando certos tipos de meios sociais, representados como potencialmente discriminatórios (Souza, Silva, Meirelles, 2013).

Pacientes com doenças transmissíveis seja hanseníase, tuberculose, HIV/AIDS, hepatites, sofre as ações do tipo restritivas de cunho direta e indireta dos profissionais de saúde que ali articulam a assistência, potencializando os sentimentos diminutivos e gerando momentos de repressão ao tratamento provido pela unidade hospitalar, a qual muitos sofrem por não possuir o devido apoio familiar, decorrente do preconceito diante da patologia (Lima et al., 2014).

Nesse sentido, alguns pacientes ficam restritos em casa, sem realizar qualquer tipo de atividade como recreação e lazer, 
decorrente de sofrer certos tipos de preconceito, seja este em convívio social, ou, intrafamiliar. As atividades de recreação e lazer proporcionam o entretenimento, a distração e o divertimento, levando a uma fuga da consciência diante do seu estado de saúde. Em pacientes hospitalizados, essa prática torna-se restrita, pois muitos ficam em estado de inércia decorrente do acometimento patológico e ansiosos nos leitos (Clementino, et al., 2011).

Com isso, cabe ao enfermeiro e toda a equipe formular um programa de interação social entre os pacientes, onde eles possam relatar as suas aflições, para que assim, o seu viver não seja apenas simbólico.

\section{Autorealização/ Autoimagem}

\section{Autoestima}

e

A Baixa autoestima foi à inferência diagnóstica mais prevalente dentro da presente necessidade. Á autoestima significa a valorização de si mesmo; amor próprio. Com isso, a mesma, torna-se um motivador poderoso de comportamento, pois quando esse motivador está em baixa, o individuo apresenta sentimentos negativos, sendo um cofator pertinente em indivíduos que tenham doenças transmissíveis (Lima et al., 2014).

Os pacientes com doenças transmissíveis exteriorizam os seus sonhos, buscando as vossas realizações, elevando assim a sua autoestima. Pois, desenham a vida de forma singela, mesmo convivendo diariamente com manifestações do tipo preconceituosa, são sonhadores, querem sempre o melhor (Courtwright \& Turner, 2010). Por isso, cabe o profissional de enfermagem, abraçar estes indivíduos com todas as suas singularidades, a qual necessita de uma socialização sem medo de sofrer qualquer tipo de barreira preconceituosa.

\section{CONCLUSÃO}

Á partir dos resultados obtidos os diagnósticos de enfermagem elencados frente as necessidade humanas básicas, psicobiológicas e psicossociais, foram: Edema periférico, Nutrição dietética prejudicada, Higiene da cavidade oral prejudicada, Padrão de higiene do couro cabeludo comprometida, Insônia, Abuso de Tabaco, Baixa autoestima, Isolamento social. A concretização e divulgação deste e de outros estudos relacionados ao tema abordado podem subsidiar os profissionais da saúde em abordagem diferenciadas, mais enfáticas e que proporcionem melhor qualidade de vida ao paciente com tuberculose. Como limitações do estudo destaca-se o fato deste ter sido realizado com uma clientela específica, a qual possui peculiaridades intrínsecas.

\section{REFERÊNCIAS}

- Alves, A. R., Lopes, C. H. A. F., Jorge, M. S. B. (2010). Significado do processo de enfermagem para enfermeiros de uma unidade de terapia intensiva: uma abordagem interacionista. Rev. Esc. Enferm. USP, 42 (4), 649-55. Recuperado de http://www.scielo.br/pdf/reeusp/v42n4/v42n4a05.pd f.

- Andrade, L. L., Nóbrega, M. M. L., Freire, M. E. M., Nóbrega, R. V. (2013). Diagnósticos de enfermagem para clientes hospitalizados em uma clínica de doenças infectocontagiosas. Rev. Esc. Enferm. USP, 47(2), 448-455. Recuperado de http://www.scielo.br/scielo.php?pid=S008062342013000200025\&script=sci_arttext.

- Araújo, A. A., Nóbrega, M. M. L., Garcia, T. R. (2013) Diagnóstico e intervenções de enfermagem para pacientes portadores de insuficiência cardíaca congestiva utilizando a CIPE ${ }^{\circledR}$. Rev. Esc. Enferm. USP, 47(2), 385-392.

- Bittencourt, G. K. G. D., Crossetti, M. G. O. (2013). Habilidades de pensamento crítico no processo diagnóstico em enfermagem. Rev. Esc. Enferm. USP, 47(2), 341-7. Recuperado de: http://www.scielo.br/pdf/reeusp/v47n2/10.pdf. 


\section{Cultura de los Cuidados}

- Brasil. (2010). Doenças infecciosas e parasitárias: guia de bolso. Secretaria de Vigilância em Saúde. Departamento de Vigilância Epidemiológica. 8 ed. Brasilia: Ministério da Saúde.

- Brasil. (2012). Conselho Nacional de Saúde. Resolução nº466 de 12 de dezembro de 2012. Aprova Diretrizes e Normas Regulamentadoras de Pesquisas envolvendo Seres Humanos. Brasilia: Ministério da Saúde.Recuperado de http://conselho.saude.gov.br/comissao/conep/resolu cao.html.

- Brasil (2013). Aids e Hepatites Virais. Departamento de DST. Brasilia: Ministério da Saúde. Recuperado de http://www.aids.gov.br/pagina/aids.

- Clementino, F. S., Martiniano, M. S., Souza, J. C., Marcolino, E. C., \& Miranda, F. A. N. (2011). Tuberculose: desvendando conflitos pessoais e sociais. Rev. Enferm. UERJ, 19(4), 638-643.

- Conselho Federal de Enfermagem. (2007). Resolução COFEN n. ${ }^{\circ}$ 311/2007. Código de ética dos profissionais de enfermagem. Recuperado de: http://se.corens.portalcofen.gov.br/codigo-de-eticaresolucao-cofen-3112007.

- Courtwright, A., \& Turner, A.N. (2010). Tuberculosis and stigmatization: pathways and interventions. Public Health Rep, 125(4), 34-42.

- Faria, J.O., \& Silva, G.A. (2013) Diagnóstico de Enfermagem em pessoas com HIV/AIDS: abordagem baseada no modelo conceitual de horta. Rev. Rene, 14(2),290-300.

- Ferraz, L.F., Viriato, A., Moura, A. (2013). Análise do diagnóstico nutricional de pacientes em assistência hospitalar de infectologia. O Mundo da Saúde, 37(3), 253-258.
- Horta, W. A. (1971). A metodologia do processo de enfermagem. Rev Bras Enf, 24(6),81-95.

- Jasen, A. K., Silveira, A. L. M., Oliveira, M. A. B., Pimenta, A.M. (2013). Desfecho terapêutico de pacientes em risco nutricional admitidos em um Hospital Universitário. Rev. Min. Enferm, 17 (3), 65157. Recuperado de http://www.reme.org.br/artigo/detalhes/679.

- Lima, L.M., Cardozo-Gonzales, R.I., Schuwartz, E., Costa, L.M., Beduhn, D.A.V., Tomberg, J. (2014). Stigma and tuberculosis: according to the view professionals of primary health care. Cuid salud, 1(1), 3-10.

- Maciel, E. L. N., Guidoni, L. M., Favero, J. L., Hadad, J. D., Molino, L. P., Jonhson, J. L. et al. (2010). Efeitos adversos causados pelo novo esquema de tratamento da tuberculose preconizado pelo Ministério da Saúde do Brasil. J Bras Pneumol, 36(2), 232-238.

- Sá, L.D., Oliveira, A.A.V., Gomes, A.L.C., Nogueira, J.Á., Villa, T.C.S., \& Collet, N. (2012). Cuidado ao doente de tuberculose na estratégia saúde da família: percepções de enfermeiras. Rev. Esc. Enferm. USP, 46(2), 356-63.

- Silva, A.P., Perelli, J.G.A., Guimarães, F.J., Mangueira, S.O., Cruz, S.L., \& Frazão, I.S. (2013). Identificação do diagnóstico de enfermagem autocontrole ineficaz da saúde em alcoolistas: um estudo descritivo. Rev. Eletr. Enf., 15(4), 932-939. Recuperado de http://dx.doi.org/10.5216/ree.v15i4.19841.

- Souza, S.S., Silva, D.M.G.V., \& Meirelles, B.H.S. (2013) Representações sociais sobre a tuberculose. Acta Paul Enferm, 23(1), 23-28. 